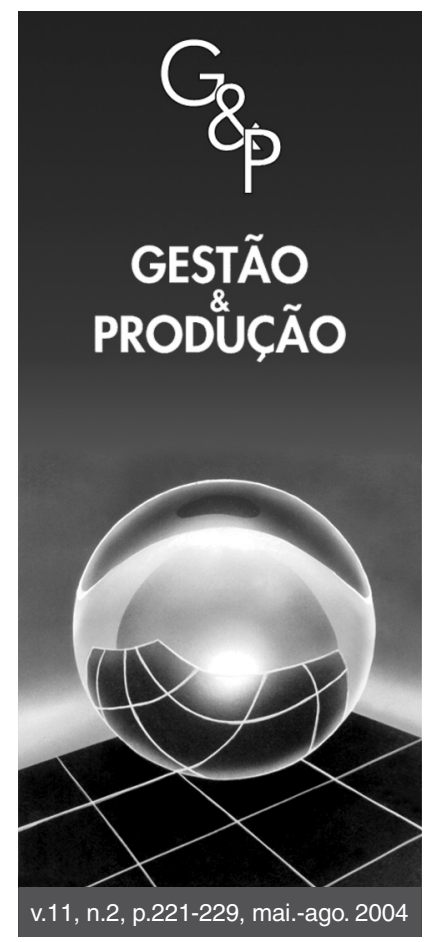

\title{
AVALIAÇÃO DE TRANSPORTADORAS DE MATERIAIS PERIGOSOS UTILIZANDO O MÉTODO ELECTRE TRI
}

\author{
Helder Gomes Costa \\ Adriana Costa Soares \\ Patricia Fernandes de Oliveira \\ Centro Tecnológico (CTC), Departamento de Engenharia de Produção (TEP), \\ Universidade Federal Fluminense, \\ Rua Passos da Pátria, 156, Bloco D, \\ CEP: 24210-240, Niterói, RJ, \\ e-mail: hgc@vm.uff.br
}

Recebido em 10/10/2002

Aceito em 30/4/2004

\section{Resumo}

Neste trabalho apresenta-se uma metodologia para a classificação e escolha de prestadores de serviço para transporte de materiais perigosos, fundamentado na metodologia de Auxílio Multicritério à Decisão-AMD. A metodologia aqui proposta apresenta características que permitem considerar a subjetividade inerente ao processo de avaliação de desempenho dos Prestadores de Serviço, diferenciando-se, assim, de outros métodos de seleção de fornecedores de serviços de transportes. As particularidades da metodologia proposta, principalmente em relação à sua aplicabilidade, foram verificadas num caso em uma empresa distribuidora de combustíveis, com rede de postos em todo o Brasil.

Palavras- chave: multicritério, MCDM, transporte.

\section{Introdução}

Conforme reportado em Carvalho e Costa (2001), todo o esforço empreendido em melhorias internas, objetivando a satisfação dos consumidores, fica comprometido quando algum elo anterior da cadeia de suprimentos falha no cumprimento de sua parte. Segundo COOPER (1997), neste ambiente, as relações entre organizações devem ser revistas, buscando-se o gerenciamento de toda a cadeia de suprimentos. Em acordo com esta ótica, uma das opções é a concretização de parcerias. Essa alternativa é cada vez mais freqüente em atividades complementares e de apoio, pois a empresa contratante pode se concentrar no seu core business. Conforme reportado em Robles e Fischmann (2001), apesar da terceirização ser um fato, não se observa neste momento de transição a formação de parcerias estratégicas.Assim sendo, é preciso fazer uma boa seleção do parceiro de negócio (prestador de serviço), examinando sua efetiva qualificação referente à execução de suas atividades para garantir o sucesso da parceria. Este processo pode gerar dúvidas e tensão devido às incertezas inerentes ao mesmo. Neste contexto, Lambert (1996) enfoca o processo de implementação e operacionalização do Gerenciamento da Cadeia de Suprimentos, destacando as questões e dificuldades associadas a este processo.

No negócio de distribuição de combustíveis, estas questões são ampliadas e intensificadas com a desregulamentação do mercado e o aparecimento de pequenas distribuidoras que conseguem atrair clientes, tornando a competição cada vez mais acirrada neste ramo de atividade. A terceirização desta distribuição é uma tendência do mercado. Como consequiência da análise de relações custo/benefício, as distribuidoras de combustíveis resolveram terceirizar a frota, pois com mais de um fornecedor, conseguem-se preços de frete mais competitivos e disponibilizam-se os ativos da empresa. A escolha de prestadores de serviço para a distribuição de combustíveis é um problema que precisa ser analisado e estudado com cuidado. Terceirizar significa passar a responsabilidade da realização de um bom serviço a outra empresa. Garantir a qualidade desse serviço é garantir a satisfação dos clientes e a imagem da empresa.

O desafio é estabelecer parcerias obtendo altos padrões nos serviços oferecidos ao cliente em aspectos como: rapidez, pontualidade, índice zero de perdas e avarias, regularidade e confiança, dentre outros. Por se tratar de transporte 
de material perigoso, a escolha da transportadora deve ser avaliada à luz de vários critérios, pois os impactos ocasionados, devido a acidentes com as cargas, podem causar danos adversos ao meio-ambiente, perdas inaceitáveis de vidas, danos irreversíveis à saúde, danos patrimoniais seguidos de uma forte comoção social, resultando em elevados custos sociais, econômicos, políticos, além de sanções legais.

\subsection{Caracterização do problema}

Neste contexto, um dos problemas principais consiste em classificar empresas distribuidoras segundo padrões pré-estabelecidos. A Figura 1 busca ilustrar este tipo de problema.

A solução deste problema envolve a avaliação de alternativas à luz de múltiplos fatores ou critérios de escolha e julgamentos subjetivos do gestor de transportes. Neste âmbito, imprecisões e incertezas são amplificadas, dificultando o estabelecimento de padrões bem definidos e eficazes, originando escolhas inadequadas que conduzem a problemas de demandas não atendidas e atrasos na entrega.

Este problema é complexo e a adoção de procedimentos empíricos ou intuitivos pode não conduzir a bons resultados. Assim, é de extrema relevância a utilização de ferramentas que possibilitem uma escolha eficaz. No entanto, apesar da existência de ferramentas de auxílio à decisão, desenvolvidas para tratar problemas envolvendo múltiplos critérios e avaliações subjetivas (denominadas ferramentas de Auxílio Multicritério à Decisão, AMD), não se observa na literatura a difusão da modelagem deste tipo de problema utilizando tais ferramentas.

\subsection{Objetivo}

Objetivando contribuir ao preenchimento desta lacuna, o presente trabalho apresenta uma metodologia alternativa,

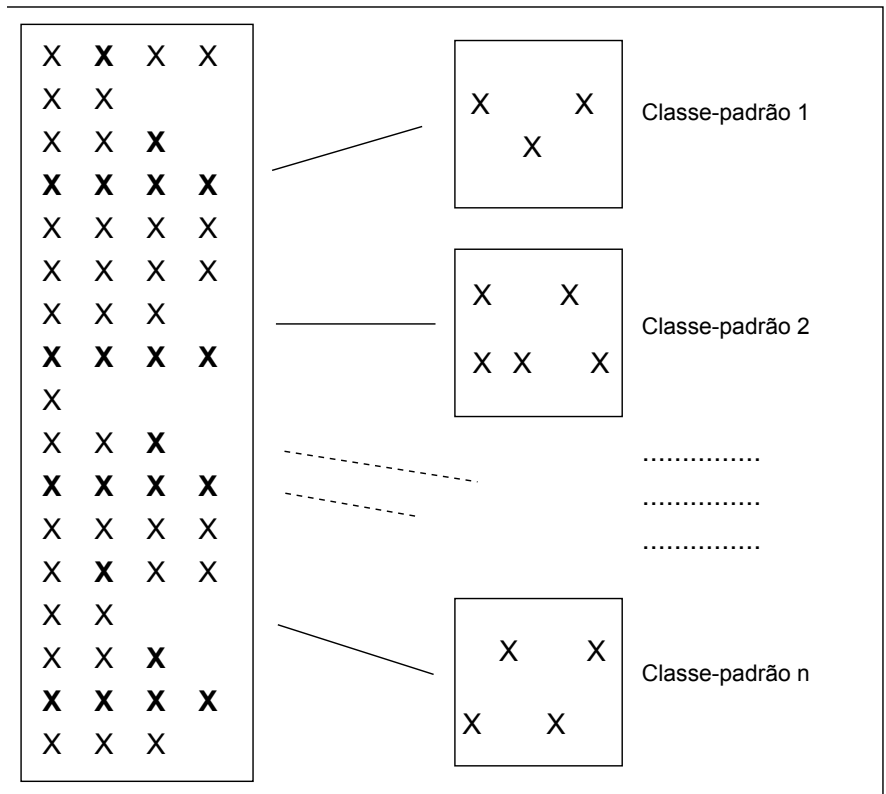

Figura 1. Problema de Classificação. (Adaptado de Mousseau et al. 1999). baseada na análise multicritério, para a classificação de empresas transportadoras de material combustível.

\section{Metodologia proposta}

A metodologia proposta neste trabalho fundamenta-se nos conceitos da AMD, mais especificamente o Método Electre Tri reportado em Yu (1992), Mousseau et al. (1999) e Rogers et al. (2000). A seguir, descrevem-se as etapas desta metodologia:

a) Identificar e caracterizar o problema. Identificar os produtos a serem transportados e as distribuidoras capazes de realizar a distribuição destes produtos;

b) Especificar os critérios. Definir os critérios a serem considerados na avaliação das distribuidoras em estudo. Este processo consiste em uma análise situacional, posicionando a distribuidora analisada quanto aos fatores que mais influenciarão seu desempenho. Conforme reportado em Azevedo e Costa (2001), a percepção destes critérios encontra-se num contexto pessoal e ambiental, sendo um processo em permanente evolução, dada a dinâmica do ambiente e da própria percepção pessoal de quem define os critérios. Assim, critérios podem ser diferentemente percebidos por diferentes analistas, devendo ser definidos por especialista(s) no problema em questão;

c) Especificar a escala para os julgamentos dos pesos de cada critério. $O$ peso indica a importância ou influência do critério no grau de competitividade da distribuidora;

d) Atribuir pesos para cada critério. Nesta etapa, estabelecem-se os pesos associados a cada critério. Estes pesos são obtidos por de julgamentos de valor, coletados junto a especialistas, com o auxílio de escala de julgamentos;

e) Especificar a escala de julgamentos dos desempenhos de cada distribuidora à luz de cada critério. Avalia-se o desempenho das distribuidoras em cada critério. É possível adotar uma escala específica para cada critério;

f) Identificar as classes de equivalência juntamente com seus respectivos limites. Neste passo, estabelecem-se as classes de desempenho que servirão de padrões para classificar as distribuidoras sob análise;

g) Estabelecer os limites de preferência ( $p$ ) e de indiferença (q) para cada critério. Estes permitem considerar a natureza imprecisa e intrínseca das avaliações do desempenho das distribuidoras à luz dos critérios considerados;

h) Estabelecer o limite de veto (v) associado a cada critério. Este limite lida com o conceito de rejeição ou veto (ou redução da credibilidade) quanto à afirmação de que uma alternativa subordina um limite de classe ( e vice-versa);

i) Emitir julgamento de valor à luz de cada critério. Emitem-se julgamentos de valor, avaliando-se o desempenho da distribuidora à luz de cada critério. Esta etapa deverá ser, preferencialmente, efetuada por especialista(s) que 
tenha $(\mathrm{m})$ conhecimento profundo sobre o comportamento das distribuidoras no transporte de combustíveis. Os avaliadores para um critério não são, necessariamente, os mesmos para os demais critérios;

j) Executar o algoritmo de classificação do ELECTRE TRI. Nesta etapa obtém-se a Classificação das Distribuidoras (CD) analisadas; e

k) Analisar os resultados obtidos pela classificação. De posse dos resultados individuais de cada distribuidora à luz dos critérios considerados, avalia-se o CD de cada alternativa, analisando-se, inclusive, o grau de credibilidade destes resultados.

\section{Exemplo de aplicação da metodologia proposta}

Objetivando ilustrar a aplicação da metodologia proposta, apresenta-se um exemplo de sua aplicação. Os dados deste exemplo foram extraídos de um caso real. A seguir, descrevem-se as etapas desta aplicação:

a) Identificar e caracterizar o problema. As áreas de negócio da organização (aqui denominada Laboratório) são: lubrificantes, químicos, gás natural e distribuição de combustíveis. Seis distribuidoras foram consideradas como alternativas para o transporte destes produtos. Estas empresas são aqui denotadas por distribuidoras X, Y, Z, R, S e T. Estas empresas desenvolvem atividades de transporte de produtos combustíveis. Para o desenvolvimento do processo de avaliação, foi criado um Comitê de Avaliação (CA) formado por: Assessores de Segurança e Transportes e Chefe de Desenvolvimento de Transporte. Além destas organizações, foi também considerada uma organização hipotética I. O uso desta organização tem por finalidade ilustrar a habilidade da metodologia em identificar possíveis Incomparabilidades ou Incoerências no modelo de classificação;

b) Especificar os critérios. Os critérios foram definidos pelo Chefe de Desenvolvimento de Transporte. Foram considerados os seguintes critérios:

- Cr1: Segurança - observam-se as condições de segurança oferecidas pela transportadora analisada, no transporte dos produtos;

- Cr2: Custo de Frete - neste critério avalia-se o preço do frete apresentado pela transportadora;

- Cr3: Nível de Serviço - avalia-se a flexibilidade da distribuidora perante flutuações da demanda;

- Cr4: Sistema de Gestão da Qualidade - analisa-se o comprometimento da distribuidora em relação à qualidade do seu serviço; e

- Cr5: Capacidade de Negociação - neste critério considera-se a flexibilidade da transportadora na negociação de reajustes de preços.

c) Especificar a escala para os julgamentos dos graus de importância (pesos) de cada critério. Com base na esca- la de Likert (1971), que foi desenvolvida para tratar com julgamentos da natureza dos presentes no problema abordado, adotou-se a escala ilustrada na Tabela 1;

d) Atribuir pesos para cada critério. A Tabela 2 ilustra os pesos dos critérios considerados. Estes pesos também foram emitidos pelo Chefe de Desenvolvimento de Transporte;

e) Especificar a escala de julgamentos dos desempenhos de cada distribuidora à luz de cada critério. As escalas de julgamento utilizadas para a avaliação do desempenho das alternativas à luz dos critérios estão ilustradas a seguir. Estas escalas foram obtidas com base na escala de Likert e por consenso do Comitê de Avaliação (Tabela 3 a 7);

f) Identificação das classes de equivalência para cada conjunto de critérios. Conforme descrito em Yu (1992) e em Mousseau et al. (1995), as classes de equivalência são definidas por limites inferiores e superiores. A Tabela 8 ilustra as classes de equivalência consideradas no presente trabalho;

g) Emitir julgamento de valor, avaliando o desempenho das alternativas à luz dos critérios. A Tabela 9 apresenta os desempenhos que foram atribuídos a cada distribuidora à luz de cada critério considerado. Estes desempenhos foram obtidos por julgamento de valor, emitidos consensualmente pelos membros do Comitê de Avaliação (CA);

h) Estabelecer o limite de preferência $(p)$, de indiferença (q) para cada critério. No presente trabalho adotaram-se os valores dos limites de preferência $(p)$ e de indiferença $(q)$ iguais a 0,0 . Estes valores foram obtidos com base na escala de julgamentos utilizada, que contém valores inteiros no intervalo de 1 a 5, e na definição dos perfis das classes - apresentada no tópico anterior. Há que se observar que:

- Qualquer valor no intervalo [0; 0,5) tem o mesmo efeito $\Rightarrow$ em função da escala de julgamentos adotada;

- $\mathrm{q} \leq \mathrm{p}<0,5 \Rightarrow$ em função das classes adotadas;

Tabela 1. Escala para julgamento da importância dos critérios.

\begin{tabular}{lc}
\hline Escala Verbal & Valor Numérico \\
\hline Extrema & 4 \\
Alta & 3 \\
Média & 2 \\
Baixa & 1 \\
Desprezível & 0 \\
\hline
\end{tabular}

Tabela 2. Pesos atribuídos aos critérios.

\begin{tabular}{ccc}
\hline Critério & & Peso \\
\hline Cr1 & Segurança & 4 \\
Cr2 & Custo & 2 \\
Cr3 & Nível de Serviço & 3 \\
Cr4 & Sistema de Gestão & 2 \\
Cr5 & Capacidade de Negociação & 2 \\
\hline
\end{tabular}


- Para o critério Segurança:

Tabela 3. Escala para julgamento de valor das transportadoras à luz da segurança.

Avaliação $\quad$ Nota

Atende de 100 a $80 \%$ dos itens da Inspeção de Segurança estabelecidos pelo Laboratório.

Atende de 79 a $60 \%$ dos itens da Inspeção de Segurança 4 estabelecidos pelo Laboratório.

Atende de 59 a $40 \%$ dos itens da Inspeção de Segurança 3 estabelecidos pelo Laboratório.

Atende de 39 a $20 \%$ dos itens da Inspeção de Segurança estabelecidos pelo Laboratório.

Atende a menos de $19 \%$ dos itens da Inspeção de Segurança estabelecidos pelo Laboratório.

- Para o critério Custos de Frete:

Tabela 4. Escala para julgamento de valor das transporadoras à luz dos Custos de Frete.

\begin{tabular}{lc}
\hline Descrição & Nota \\
\hline Custo de 10\% abaixo do custo estabelecido pelo Laboratório. & 5 \\
Custo de 5\% abaixo do custo estabelecido pelo Laboratório. & 4 \\
Custo estabelecido pelo Laboratório. & 3 \\
Custo de 5\% acima do custo estabelecido pelo Laboratório. & 2 \\
Custo de 10\% acima do custo estabelecido pelo Laboratório. & 1 \\
\hline
\end{tabular}

- Sistemas de Gestão da Qualidade:

Tabela 5. Escala para julgamento de valor das transportadoras à luz do Nível de Serviços.

\begin{tabular}{lc}
\hline Descrição & Nota \\
\hline Atende até $20 \%$ a variação de demanda. & 5 \\
Atende até 15\% a variação de demanda. & 4 \\
Atende até 10\% a variação de demanda. & 3 \\
Atende até 5\% a variação de demanda. & 2 \\
Não atende a variação de demanda. & 1 \\
\hline
\end{tabular}

- Para o critério Nível de Serviços:

Tabela 6. Escala para julgamento de valor das transportadoras à luz do Sistema de Gestão.

\begin{tabular}{lc}
\hline Descrição & Nota \\
\hline $\begin{array}{l}\text { É certificado pela norma ISO 9000, tendo sido certificado } \\
\text { mais de uma vez. }\end{array}$ & 5 \\
É certificado pela norma ISO 9000. & 4 \\
$\begin{array}{l}\text { Não é certificado, mas possui um sistema que engloba os } \\
\text { padrões da ABNT. }\end{array}$ & 3 \\
Não estabelece claramente os padrões de qualidade. & 2 \\
Não há referência de padrões de qualidade no serviço. & 1 \\
\hline
\end{tabular}

- Capacidade de Negociação:

Tabela 7. Escala para julgamento à luz da Capacidade de Negociação.

\begin{tabular}{lc}
\hline Descrição & Nota \\
\hline Altamente flexível e aberta a negociações. & 5 \\
Flexível e aberta a negociações. & 4 \\
Há negociação, mas há um certo desgaste nas relações. & 3 \\
Negociação é demorada e o desgaste é intenso. & 2 \\
Não são abertos a negociações. & 1 \\
\hline
\end{tabular}

Tabela 8. Classes de equivalência e seus limites superiores e inferiores.

\begin{tabular}{clcc}
\hline Classes & Descrição das classes & $\begin{array}{c}\text { Limite } \\
\text { Superior }\end{array}$ & $\begin{array}{c}\text { Limite } \\
\text { Inferior }\end{array}$ \\
\hline A & Padrão de serviço MUITO BOM & - & 4,5 \\
B & Padrão de serviço BOM & 4,5 & 3,5 \\
C & Padrão de serviço REGULAR & 3,5 & 2,5 \\
D & Padrão de serviço RUIM & 2,5 & 1,5 \\
E & Padrão de serviço MUITO RUIM & 1,5 & - \\
\hline
\end{tabular}

Tabela 9. Desempenho de cada distribuidora à luz de cada critério.

\begin{tabular}{llcccccc}
\hline \multirow{2}{*}{ Critério } & & \multicolumn{7}{c}{ Desempenho das Distribuidoras } \\
& & R & S & T & X & Y & Z \\
\hline Cr1 & Segurança & 4 & 5 & 4 & 2 & 4 & 3 \\
Cr2 & Custo & 4 & 4 & 4 & 5 & 2 & 4 \\
Cr3 & Nível de Serviço & 3 & 4 & 3 & 2 & 3 & 3 \\
Cr4 & Sistema de Gestão & 4 & 3 & 3 & 3 & 4 & 3 \\
Cr5 & Capacidade de Negociação & 2 & 5 & 2 & 3 & 2 & 4 \\
\hline
\end{tabular}

i) Estabelecer o limite de veto (v) para cada critério. Quanto ao veto, após apresentação do conceito de veto ao Comitê de Avaliação (CA) o mesmo decidiu por não considerar o veto na modelagem do problema. A justificativa apresentada pelos membros do CA é de que as empresas participantes no processo de classificação já passavam por um processo prévio de seleção

j) Executar o algoritmo de classificação do ELECTRE TRI. A Tabela 10 ilustra os resultados obtidos pela execução do algoritmo de classificação do ELECTRE TRI. Conforme descrito no anexo, no ELECTRE TRI comparam-se as alternativas aos perfis que delimitam as classes de referência, definidas no tópico (f), fornecendo duas classificações:

- classificação mais exigente (pessimista); e

- classificação menos exigente (otimista).

Neste modelagem, fez-se uma análise de sensibilidade dos resultados em função de diferentes valores para o nível de corte $(\lambda)$ dentre um intervalo possível $[0,5 ; 1]$. Os resul- 
tados obtidos destas análises estão apresentados nas Tabelas 10 e 11. Mais especificamente, a Tabela 10 apresenta os resultados obtidos para $\lambda \varepsilon(0,5 ; 0,85)$ e a Tabela 11 apresenta os resultados obtidos para $\lambda \varepsilon[0,85 ; 1,0]$.

Quando as duas classificações (mais exigente e menos exigente) convergem, significa que o sistema construído foi capaz de restabelecer as comparações das alternativas aos perfis. A divergência entre estas classificações para uma das alternativas indica a incapacidade do sistema de comparar esta alternativa a pelo menos um dos limites de Classes. A análise dos resultados obtidos é apresentada no próximo tópico.

k) Analisar os resultados obtidos pela classificação. Estes resultados ilustram a classificação obtida por cada distribuidora, quando comparada às classes de equivalência. Estas classes de equivalência se comportam como padrões de referência. Analisando os resultados expostos na Tabela 10 e 11, pode-se observar que:

- Para níveis de credibilidade menores do que 0,85 ( $\lambda$ $<0,85)$ não foram identificadas incomparabilidades. Isto indica que o sistema mostrou-se coerente na busca da classificação das alternativas para estes níveis de credibilidade. Neste caso, os resultados indicam que a alternativa $S$ é a alternativa mais indicada, estando classificada na Classe A. Em um segundo plano surgem as alternativas $\mathrm{R}, \mathrm{T}$ e Z, classificadas na Classe $\mathrm{B}$. As piores alternativas foram as alternativa $\mathrm{X}$ e $\mathrm{Y}$, classificadas nas Classes D e C, respectivamente.

- Para níveis de credibilidade menores ou iguais a 1,0 e maiores ou iguais a $0,85(\lambda \varepsilon[0,85 ; 1.00])$ somente a alternativa $X$ apresentou divergência de classificações:

Tabela 10. Classificação (pelo ELECTRE TRI) das prestadoras de serviço, $\lambda \varepsilon[0,5 ; 0,85]$.

\begin{tabular}{clcc}
\hline Distribuidora & $\begin{array}{c}\text { Classificação } \\
\text { mais exigente }\end{array}$ & $\begin{array}{c}\text { Classificação } \\
\text { menos exigente }\end{array}$ & $\begin{array}{c}\text { Classificação } \\
\text { final }\end{array}$ \\
\hline $\mathrm{R}$ & Classe B & Classe B & Classe B \\
$\mathrm{S}$ & Classe A & Classe A & Classe A \\
$\mathrm{T}$ & Classe B & Classe B & Classe B \\
$\mathrm{X}$ & Classe D & Classe D & Classe D \\
$\mathrm{Y}$ & Classe C & Classe C & Classe C \\
$\mathrm{Z}$ & Classe B & Classe B & Classe B \\
\hline
\end{tabular}

Tabela 11. Classificação (pelo ELECTRE TRI ) das prestadoras de serviço, $\lambda \varepsilon[0,85 ; 1,0]$.

\begin{tabular}{clcc}
\hline Distribuidora & $\begin{array}{c}\text { Classificação } \\
\text { mais exigente }\end{array}$ & $\begin{array}{c}\text { Classificação } \\
\text { menos exigente }\end{array}$ & $\begin{array}{c}\text { Classificação } \\
\text { final }\end{array}$ \\
\hline $\mathrm{R}$ & Classe B & Classe B & Classe B \\
$\mathrm{S}$ & Classe A & Classe A & Classe A \\
$\mathrm{T}$ & Classe B & Classe B & Classe B \\
$\mathrm{X}$ & Classe D & Classe D & Classe D \\
$\mathrm{Y}$ & Classe C & Classe C & Classe C \\
$\mathrm{Z}$ & Classe B & Classe B & Classe B \\
\hline
\end{tabular}

Classe BOM, sob a ótica otimista; e, Classe RUIM sob a ótica pessimista. Esta dupla classificação ilustra uma incomparabilidade. Esta incomparabilidade está associada a uma incoerência do sistema de classificação, quando busca comparar a alternativa $\mathrm{X}$ às classes de equivalência. Isto significa que o sistema de classificação precisa ser reavaliado, caso seja estritamente necessária uma convergência entre as classificações otimista e pessimista para esta alternativa. Em geral, as fontes de incomparabilidade estão associadas:

- Ao modelo construído;

- Às escalas de julgamento; e

- Aos julgamentos de valor considerados na avaliação de desempenho das alternativas à luz dos critérios.

Nos dois primeiros casos, a incomparabilidade é devida a uma falha na modelagem e, em geral, é observada em mais de uma alternativa. Para a eliminação deste tipo de incomparabilidade é necessário rever os parâmetros do modelo construído. No terceiro caso, a incomparabilidade é eliminada por de um processo de revisão dos julgamentos referentes à alternativa.

Caso seja crucial obter a classificação da Distribuidora X, a despeito de divergências ocorridas entre as classificações pessimista e otimista, o classificador pode adotar uma das duas classificações de acordo com o seu perfil: (mais exigente ou menos exigente). Este procedimento também é adotado em outras situações de decisão em que se classifica o perfil do decisor em otimista ou pessimista, como na Teoria dos Jogos. Mais ainda, no caso do ELECTRE TRI, uma divergência entre estas classificações indica uma incapacidade do sistema em comparar o elemento sendo classificado a pelo menos um dos perfis das classes de equivalência utilizadas. Esta incapacidade pode ser causada pelo avaliador, pelo modelo de classificação (incluindo o conjunto de critérios) ou pelo sistema de coleta de dados (incluindo as escalas utilizadas).

Observa-se que não foram encontradas incomparabilidades (mesmo para credibilidade $=1,0$ ) com as demais alternativas do caso realizado, indicando um alto grau de coerência do modelo para classificação destas alternativas. Isto significa que o sistema mostrou-se consistente no processo de classificação desta distribuidora. A incomparabilidade observada para a Empresa X possibilitou ilustrar a capacidade do método detectar "ruídos" (leia-se incomparabilidades) que outros métodos (que fazem uso da média ponderada) não são capazes de captar.

A Tabela 12 ilustra a classificação que seria obtida caso usássemos os mesmos limites de classe ilustrados na Tabela 8 , porém utilizando a média ponderada como instrumento de agregação para obtenção da classificação final.

Este resultado mostra que a classificação pela média ponderada agrupou na mesma classe as alternativas R, S, T, X: Classe B. Este fato ocorreu devido ao princípio de agregação presente na função média ponderada, diferentemente do princípio de superação presente nos métodos da Família 
Tabela 12. Classificação das prestadoras de serviço utilizando a média ponderada.

\begin{tabular}{cc}
\hline Distribuidora & Classificação por média ponderada \\
\hline $\mathrm{R}$ & Classe B \\
$\mathrm{S}$ & Classe B \\
$\mathrm{T}$ & Classe B \\
$\mathrm{X}$ & Classe B \\
$\mathrm{Y}$ & Classe C \\
$\mathrm{Z}$ & Classe C \\
\hline
\end{tabular}

ELECTRE. Segundo este princípio, para que uma alternativa (a) supere uma outra alternativa (b) é necessário que (a) tenha desempenho pelo menos tão bom quanto o de (b) em um determinado número de critérios.

Para se estabelecer bem a diferença entre o emprego da média ponderada e dos métodos de superação, pode-se fazer uma analogia com o que acontece em uma partida de voleibol no confronto entre os times A e B. Se no primeiro 'set', B ganha de A por 25 a 0 ; porém, nos demais três 'sets', A ganha de B por 25 a 20, podemos ter duas análises:

(i) Usar a média ponderada para obter o resultado final. Neste caso B seria o vencedor da partida por 85 a 75 ; e

(ii) Usar o número de 'sets' para definir o vencedor. Neste caso A seria o vencedor por 3 a 1 . O princípio fundamental dos métodos de superação pode ser considerado semelhante a esta $2^{\mathrm{a}}$ abordagem, se considerarmos que cada 'set' equivale a um dos critérios da análise multicritério.

\section{Conclusões}

Em geral, os julgamentos dependem da avaliação de diversas variáveis simultaneamente e de interpretações pessoais múltiplas, que variam de acordo com a experiência/ preferência do avaliador. O mesmo ocorre em situações de decisões de seleção de transportadoras. A metodologia proposta neste trabalho aplica-se diretamente a casos com estas características. Por meio da sua utilização, os decisores (executivos) obterão informações consistentes sobre o grau de competitividade da distribuidora, reduzindo a incidência e a intensidade das inconsistências na sua tomada de decisão.
Observa-se que não foram encontradas incomparabilidades, no Estudo de Caso realizado, para classificação das Distribuidoras $\mathrm{R}, \mathrm{S}, \mathrm{T}, \mathrm{Y}, \mathrm{Z} \operatorname{com} \lambda \varepsilon[0,5 ; 1,0]$. Isto significa que o sistema mostrou uma excelente consistência no processo de classificação destas distribuidoras analisadas. No entanto, para o caso da Distribuidora $\mathrm{X}$, o sistema não se mostrou adequado para a classificação da Distribuidora $X$ nas classes definidas. Este fato chama a atenção para a análise da alternativa $X$, mostrando que esta não mantém regularidade de desempenho. Ou seja: seu desempenho oscila em diferentes classes, dificultando a sua classificação.

Este fato é didaticamente positivo, pois ilustra a capacidade do método em detectar "ruídos" que outros métodos (tais como aqueles que fazem uso da média ponderada) não são capazes de captar.

A utilização desta metodologia permite identificar incomparabilidades ou inconsistências não detectáveis pelos métodos que se baseiam em médias ponderadas. Outra importante diferença em relação aos métodos tradicionais é que a metodologia aqui proposta classifica, em níveis mais altos, aquelas distribuidoras que tem um melhor desempenho em um maior número de critérios, independente do valor alcançado pelas distribuidoras na média ponderada. Por disponibilizar resultados mais refinados, a metodologia pode ser utilizada para melhor orientar a escolha da distribuidora, pois a classificação encontrada reflete o estilo de atuação da distribuidora desejado pela contratante.

Apesar da aplicação desta metodologia depender do uso de um método não muito difundido no meio organizacional, o Método ELECTRE TRI, este não é um fator prejudicial em sua aplicabilidade.

Outro aspecto relevante é a originalidade da metodologia proposta, que considera a subjetividade inerente aos avaliadores e que está fundamentada em algoritmos matemáticos que aumentam a credibilidade dos resultados finais.

Para futuros desenvolvimentos, sugere-se a resolução deste problema por outros métodos decisórios e de classificação à intercomparação dos resultados obtidos. Dentre estes outros métodos, sugere-se empregar o AHP, O MACBETH, PROMETHÉE, REAL OPTION. Além destes métodos, poderá ser investigada, também, a modelagem deste problema por Redes de Neurônios Artificiais (NNW), Conjuntos Aproximativos (ROUGH SETS) e Lógica Nebulosa (Fuzzy Logic).

\section{Referências Bibliográficas}

AZEVEDO, M. C. de; COSTA, H. G. Metodologia para o diagnóstico estratégico. In: CONFERENCIA INTERNACIONAL DE CIENCIAS EMPRESARIALES. Anais da ... Santa Clara, Cuba: Universidad Marta Abreu, 2000. $1 \mathrm{CD}-\mathrm{ROM}$.

CARVALHO, R. A.; COSTA, H.G. Tendências no fornecimen- to de materiais. In: XXI ENEGEP (Encontro Nacional de Engenharia de Produção) / VII ICIE (International Congress of Industrial Engineers). Anais do ... / Proceedings of ... Salvador, BA, Brasil, 2001. 1 CD-ROM.

COOPER, M. C.; LAMBERT, D. M.; PAGH, J. D. Supply chain management: more than a new name for logistics. The 
International Journal of Logistics Management. (8), 1, U.S.A. 1997.

LAMBERT, D. M.; EMMELHAINZ. M. A.; GARDNER, J. T. Developing and Implementing Supply Chain Partnerships. The International Journal of Logistics Management. (7), 2, U.S.A. 1996.

LIKERT, R. Novos padrões de administração. São Paulo: Editora Pioneira, 1971.

MOSSEAU, V.; SLOWINSKI, R.; ZIELNIEWICZ, P. ELECTRE TRI 2.0a. Methodological Guide and User's Manual. Document Du Lamsade. France: Université Paris - Dauphine., fev. 1999.

ROBLES, L.T., FISCHMANN, A. Características do Relacionamento entre Montadoras e Operadores Logísticos e a
Prestação de Serviços de Logística Integrada na Indústria Automobilística no Brasil. In: XXV ENAMPAD (Encontro Nacional da Associação Nacional de Programas de Pós-graduação em Administração). Anais do ... Campinas, SP, Brasil: ANPAD, 2001. 1 CD-ROM.

ROGERS, M., BRUEN, M., MAYSTRE, L. ELECTRE and Decision Support: Methods and Applications in Engineering and Infrastructure Investment. USA: Kluwer Academic Publishers, 2000.

ROY, B., BOYSSOU, D. Aid Multicritère à la decision. Paris: Econômica, 1993.

YU, W. ELECTRE TRI Aspects Methodologiques et Guide d'Utilisation. Document Du Lamsade. France: Université Paris - Dauphine, 1992.

\section{THE ELETRE TRI METHOD APPLIED TO THE EVALUATION OF COMPANIES TRANSPORTING HAZARDOUS MATERIALS}

\section{Abstract}

This article proposes a method for the classification and selection of hazardous materials transporters based on Multicriteria Decision Making (MCDM) concepts. This approach involves characteristics that allow the subjectivity inherent to the process of evaluating the performance of Service Companies to be considered, thus differentiating it from other transport services supplier selection methods.

The particularities of the proposed methodology, particularly insofar as its applicability is concerned, were assessed by means of a case study involving a fuel distribution company which owns gas stations throughout the country.

Keywords: multicriteria, MCDM, transport.

\section{ANEXO: Descrição do método ELETRE TRI}

O Método ELECTRE TRI reportado em YU (1992), Roy e Boyssou (1993) e Mousseau et al. (1999), pertence à família ELECTRE e caracteriza-se por tratar de problemas específicos de classificação ordenada. Ou seja: dado um conjunto de $\mathbf{A}=\{\mathrm{a}, \mathrm{b}, \mathrm{c}, .$.$\} de alternativas, associa-as a um conjunto$ de classes ordenadas $\mathbf{C}=\{\mathrm{C} 1, \mathrm{C} 2, \ldots . . \mathrm{Cn}\}$, considerando o desempenho de $\underline{\mathrm{A}}$ à luz de um conjunto de critérios $\mathbf{F}=\left\{\mathrm{g}_{1}\right.$, $\left.\mathrm{g}_{2}, \ldots, \mathrm{g}_{\mathrm{m}}\right\}$. As classes são delimitadas por limites superiores $\mathrm{e}$ limites inferiores, conforme ilustrado na Figura A.1.

Este método integra funções específicas que dão suporte ao decisor no processo de preferência e reduzem o esforço cognitivo requerido na fase de modelagem. O ELECTRE TRI classifica as alternativas seguindo dois passos consecutivos: construção de uma relação de subordinação $S$, que caracteriza como as alternativas são comparadas aos limites das classes; e, exploração (através de procedimentos de classificação) da relação $S$.

\section{Relação de subordinação no ELECTRE TRI}

A relação de subordinação é construída para tornar possível a comparação de uma alternativa a com um limite padrão bh. A afirmação de que $a S b_{h}$ significa que " $a$ é ao menos tão boa quanto $b h$ ". Na validação da afirmação $a S b_{h}$ (ou $b_{h} S a$ ), devem-se verificar duas condições:

- Concordância: para que $a S b_{h}$ (ou $b_{h} S a$ ) seja aceita, uma maioria suficiente de critérios deve ser a favor desta afirmação; e

- Não-discordância: quando na condição de concordância esperada, nenhum dos critérios na minoria deve se opor à afirmação $a S b h$ (ou $b_{h} S a$ ).

Dois tipos de parâmetros associados aos de critérios intervêm na construção de $S$ :

- O conjunto de coeficientes dos pesos ou importância $(k l$, $k 2, \ldots, k m$ ), usado no teste de concordância quando se computa a importância relativa da união dos critérios que são a favor da afirmação $a S b_{h}$; e

- $O$ conjunto de limites de veto $\left(v 1\left(b_{h}\right), v 2\left(b_{h}\right), \ldots, v m\left(b_{h}\right)\right), \forall h$ 


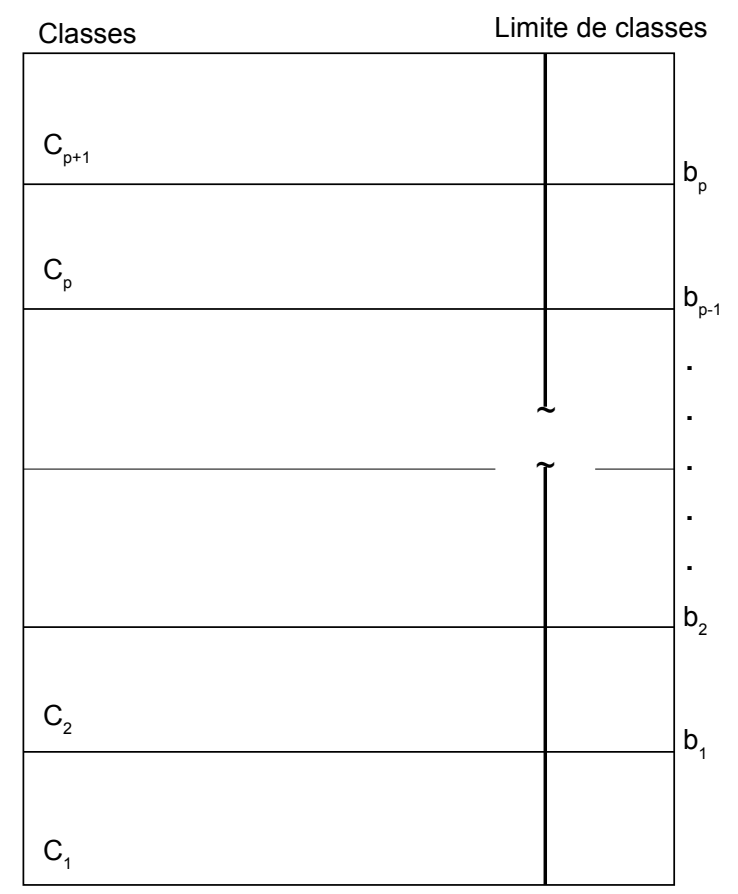

Figura A1. Classes de equivalência no ELECTRE TRI

$\epsilon B$, usado no teste de discordância $v_{j}\left(b_{h}\right)$ representa a menor diferença $g_{j}\left(b_{h}\right)-g_{j}(a)$ incompatível com a afirmação $a S b h$.

Os seguintes passos são seguidos na obtenção desta relação :

- Computar o índice de concordância parcial $c_{j}\left(a, b_{h}\right)$ e $c_{j}\left(b_{h}, a\right)$;

- Computar o índice de concordância geral $c\left(a, b_{h}\right)$;

- Computar o índice de discordância parcial $d_{j}\left(a, b_{h}\right)$ e $d_{j}\left(b_{h}, a\right)$;

- Computar a relação de subordinação fuzzy conforme o índice de credibilidade $\sigma\left(a, b_{h}\right)$; e

- Determinar um corte $\lambda$ da relação fuzzy para obter uma relação de subordinação. Isto é: Se $\sigma\left(a, b_{h}\right) \geq \lambda \Rightarrow a S b_{h}$.

$\mathrm{O}$ índice de concordância parcial $c_{j}\left(a, b_{h}\right)$ expressa até que ponto a afirmação " $a$ " é ao menos tão boa quanto $b_{h}$ considerando o critério " $g$ " é válida. Este índice é computado pela seguinte expressão:

- Quando $g_{j}$ tem uma direção de preferência crescente, $c_{j}(a$, $b_{h}$ ) é computado como se segue:

- Se $g_{j}(a) \leq g_{j}\left(b_{h}\right)-p_{j}\left(b_{h}\right)$, então $c_{j}\left(a, b_{h}\right)=0$

- Se $g_{j}\left(b_{h}\right)-p_{j}\left(b_{h}\right)<g_{j}(a) \leq g_{j}\left(b_{h}\right)-q_{j}\left(b_{h}\right)$, então $c_{j}\left(a, b_{h}\right)=\left[g_{j}(a)-g_{j}\left(b_{h}\right)+p_{j}\left(b_{h}\right)\right]$

$$
\left[p_{j}\left(b_{h}\right)-q_{j}\left(b_{h}\right)\right]
$$

- Se $g_{j}\left(b_{h}\right)-q_{j}\left(b_{h}\right)<g_{j}(a)$, então $c_{j}\left(a, b_{h}\right)=1$

- Quando $g_{j}$ tem uma direção de preferência decrescente, $c_{j}\left(a, b_{h}\right)$ é computado como se segue:

- Se $g_{j}(a) \geq g_{j}\left(b_{h}\right)+p_{j}\left(b_{h}\right)$, então $c_{j}\left(a, b_{h}\right)=0$

$-\operatorname{Se} g_{j}\left(b_{h}\right)+q_{j}\left(b_{h}\right) \leq g_{j}(a) \leq g_{j}\left(b_{h}\right)+p_{j}\left(b_{h}\right)$, então $c_{j}\left(a, b_{h}\right)=\left[g_{j}\left(b_{h}\right)-g_{j}(a)+p_{j}\left(b_{h}\right)\right]$

$$
\left[\mathrm{p}_{\mathrm{j}}\left(\mathrm{b}_{\mathrm{h}}\right)-\mathrm{q}_{\mathrm{j}}\left(\mathrm{b}_{\mathrm{h}}\right)\right]
$$

- Se $g_{j}\left(b_{h}\right)+q_{j}\left(b_{h}\right)>g_{j}(a)$, então $c_{j}\left(a, b_{h}\right)=1$

$\mathrm{O}$ índice de concordância global $c_{\mathrm{j}}\left(b_{h}, a\right)$ expressa até que ponto as avaliações de a e bh, em todos os critérios, estão de acordo com a afirmação " $a$ subordina $b_{h}$ ":

$$
c_{j}\left(a, b_{h}\right)=\left[\Sigma_{j \in F} k_{j} c_{j}\left(a, b_{h}\right)\right] / \Sigma_{j \in F} k_{j}
$$

O índice de discordância parcial $d_{j}\left(a, b_{h}\right)$ expressa até que ponto o critério $g_{j}$ se opõe à afirmação " $a$ é ao menos tão boa quanto $b_{h}$ ", isto é, “ $a$ subordina $b_{h}$ ”. Um critério $g_{j}$ é considerado discordante com a afirmação " $a$ subordina $b_{h}$ " se, neste critério, $b_{h}$ é preferida à $a$. Neste caso de preferência crescente, o critério $g_{j}$ se opõe a um veto quando a diferença $g_{j}\left(b_{h}\right)-g_{j}(a)$ excede ao limite de veto $v_{j}\left(b_{h}\right)$.

- Quando $g_{j}$ tem uma direção de preferência crescente, $d_{j}(a$, $b_{h}$ ) é computado como se segue:

- Se $g_{j}(a)>g_{j}\left(b_{h}\right)-p_{j}\left(b_{h}\right)$, então $d_{j}\left(a, b_{h}\right)=0$

- Se $g_{j}\left(b_{h}\right)-v_{j}\left(b_{h}\right)<g_{j}(a) \leq g_{j}\left(b_{h}\right)-p_{j}\left(b_{h}\right)$, então $d_{j}\left(a, b_{h}\right)=\left[g_{j}\left(b_{h}\right)-g_{j}(a)-p_{j}\left(b_{h}\right)\right]$ $\left[v_{j}\left(b_{h}\right)-p_{j}\left(b_{h}\right)\right]$

- Se $g_{j}\left(b_{h}\right)-v_{j}\left(b_{h}\right) \geq q_{j}(a)$, então $d_{j}\left(a, b_{h}\right)=1$

- Quando $g_{j}$ tem uma direção de preferência decrescente, $d_{j}\left(a, b_{h}\right)$ é computado como se segue:

- Se $g_{j}(a) \leq g_{j}\left(b_{h}\right)+p_{j}\left(b_{h}\right)$, então $d_{j}\left(a, b_{h}\right)=0$

$-\operatorname{Se~}_{\mathrm{j}}\left(b_{\mathrm{h}}\right)+\mathrm{p}_{\mathrm{j}}\left(\mathrm{b}_{\mathrm{h}}\right)<\mathrm{g}_{\mathrm{j}}(\mathrm{a}) \leq \mathrm{g}_{\mathrm{j}}\left(\mathrm{b}_{\mathrm{h}}\right)+\mathrm{v}_{\mathrm{j}}\left(\mathrm{b}_{\mathrm{h}}\right)$,

então $d_{j}\left(a, b_{h}\right)=\left[g_{j}(a)-g_{j}\left(b_{h}\right)-p_{j}\left(b_{h}\right)\right]$

$$
\left[\mathrm{v}_{\mathrm{j}}\left(\mathrm{b}_{\mathrm{h}}\right)-\mathrm{p}_{\mathrm{j}}\left(\mathrm{b}_{\mathrm{h}}\right)\right]
$$

- Se $g_{j}\left(b_{h}\right)+v_{j}\left(b_{h}\right)<g_{j}(a)$, então $d_{j}\left(b_{h}, a\right)=1$

O grau de credibilidade da relação de subordinação $\sigma\left(a, b_{h}\right)$ expressa até que ponto " $a$ subordina $b_{h}$ " de acordo com o índice de concordância global $c_{j}\left(a, b_{h}\right)$ e com o índice de discordância $d_{j}\left(a, b_{h}\right), \forall \forall_{j \in F}$. Calcula-se o índice de credibilidade $\sigma\left(a, b_{h}\right)$ e $\sigma\left(b_{h}, a\right)$ somando-se os valores estabelecidos na relação de subordinação.

O cálculo do índice de credibilidade $\sigma\left(a, b_{h}\right)$ é de acordo com os seguintes princípios:

1. quando nenhum critério for discordante, a credibilidade da relação de subordinação $\sigma\left(a, b_{h}\right)$ é igual ao índice de concordância $\sigma\left(a, b_{h}\right)$;

2. quando um critério discordante se opõe ao veto para a afirmação " $a$ subordina $b_{h}$ " (i. é, $d_{j}\left(a, b_{h}\right)=1$ ), então o índice de credibilidade $\sigma\left(a, b_{h}\right)$ torna-se nulo (a afirmação " $a$ subordina $b_{h}$ " não é totalmente acreditável); e

3. quando um critério discordante é tal como $c\left(a, b_{h}\right)<d_{j}$ $\left(a, b_{h}\right)<1$, o índice de credibilidade $\sigma\left(a, b_{h}\right)$ torna-se mais baixo do que o índice de concordância $c\left(a, b_{h}\right)$, sendo justo o efeito de oposição deste critério.

A conclusão destes princípios é de que o índice de credibilidade $\sigma\left(a, b_{h}\right)$ corresponde ao índice de concordância fraca, por um eventual efeito de veto. Mais precisamente, o valor $\sigma\left(a, b_{h}\right)$ é calculado como se segue $\left(\sigma\left(b_{h}, a\right)\right.$ é calculado similarmente):

$$
\begin{array}{r}
\sigma\left(a, b_{h}\right)=c\left(a, b_{h}\right) \prod_{j \in \bar{F}} 1-d_{j}\left(a, b_{h}\right), \\
1-c\left(a, b_{h}\right)
\end{array}
$$$$
\text { onde } \overline{\mathrm{F}}=\left\{\mathrm{j} \in \mathrm{F} / \mathrm{d}_{\mathrm{j}}\left(\mathrm{a}, \mathrm{b}_{\mathrm{h}}\right)>\mathrm{c}\left(\mathrm{a}, \mathrm{b}_{\mathrm{h}}\right)\right\}
$$

A tradução de uma relação de subordinação fuzzy obtida 
entre uma relação de subordinação $\mathrm{S}$ é feita sobre o significado de um corte- $\lambda$ ( $\lambda$ é chamado de nível de corte). $\lambda$ é considerado como o menor valor do índice de credibilidade compatível com a afirmação de que " $a$ subordina $b_{h}$ ", i. é, $\sigma\left(a, b_{h}\right) \geq \lambda \Rightarrow a S b_{h}$. Assim, são definidas relações binárias preferência ( $>$ ), indiferença (I) e incomparabilidade (R):

- $\mathrm{aIb}_{\mathrm{h}} \Leftrightarrow \mathrm{aSb}_{\mathrm{h}}$ é $\mathrm{b}_{\mathrm{h}} \mathrm{Sa}$;

- $\mathrm{a}>\mathrm{b}_{\mathrm{h}} \Leftrightarrow \mathrm{aSb}_{\mathrm{h}}$ e não $\mathrm{b}_{\mathrm{h}} \mathrm{Sa}$

$\bullet a<b_{h} \Leftrightarrow$ não $a b_{h}$ e $b_{h} S a ; e$

$\bullet \mathrm{aRb}_{\mathrm{h}} \Leftrightarrow$ não $\mathrm{aSb}_{\mathrm{h}}$ e não $\mathrm{b}_{\mathrm{h}} \mathrm{as}$.

\section{Procedimentos de classificação}

A regra do procedimento de exploração é realizada para analisar o modo em que uma alternativa a é comparada com os limites padrão determinados para a classe na qual a deve ser enquadrada. Dois procedimentos de classificação são avaliados.

$1^{\circ}$ ) O procedimento de classificação pessimista (ou conjuntivo) é descrito a seguir:

1. Comparar $a$ sucessivamente com $b_{i}$, para i $=\mathrm{p}, \mathrm{p}-1, \ldots, 0$.

2. Que $b_{h}$ seja o primeiro limite padrão tal que $a S b_{h}$, classificando $a$ para a Classe $C_{h+1}\left(\mathrm{a} \rightarrow \mathrm{C}_{\mathrm{h}+1}\right)$.

Se $b_{h-1}$ e $b_{h}$ denotam o limite superior e inferior da Classe $C_{h}$, o procedimento pessimista classifica a alternativa $a$ para a mais alta Classe $C_{h}$ tal que $a$ subordine $b_{h-1}$, isto é, $a S b_{h-1}$. Quando se utiliza este procedimento com $\lambda=1$, uma alternativa $a$ pode ser enquadrada na Classe $C_{h}$ somente se $g_{j}(a)$ for igual ou exceder $g_{j}\left(b_{h-1}\right)$ (pela soma dos limites) para cada critério (regra conjuntiva). Quando $\lambda$ decresce, o caráter conjuntivo desta regra é fraco.

$2^{\circ}$ ) O procedimento de classificação otimista (ou disjuntivo) é descrito a seguir:
1. Comparar $a$ sucessivamente com $b_{i}, \mathrm{i}=1,2, \ldots, \mathrm{p}$; e

2. Que $b_{h}$ seja o primeiro limite padrão tal que $b_{h}>a$, classificando $a$ na Classe $C_{h}\left(\mathrm{a} \rightarrow \mathrm{C}_{\mathrm{h}}\right)$.

$\mathrm{O}$ procedimento otimista (ou disjuntivo) classifica $a$ para a mais baixa Classe $C_{h}$ para a qual o limite superior de $b_{h}$ é preferível a $a$, i. é, $b_{h}>a$. Quando se utiliza este procedimento $\operatorname{com} \lambda=1$, uma alternativa $a$ pode ser classificada na Classe $C_{h}$ quando $g_{j}\left(b_{h}\right)$ exceder $g_{j}(a)$ (pela soma dos limites) ao menos para um critério (regra disjuntiva). Quando $\lambda$ decresce, o caráter disjuntivo desta regra é fraco.

$3^{\circ}$ ) Comparação entre os dois procedimentos de classificação:

Sendo estes dois procedimentos diferentes, conseqüentemente, pode ocorrer a classificação de algumas alternativas em diferentes classes. O exemplo seguinte explica, num nível teórico, a razão da possibilidade de divergência dos resultados de classificação.

Suponha-se que uma alternativa $a$ é classificada em $C_{i}$ e $C_{j}$ pelas regras de classificação pessimista e otimista, respectivamente. Espera-se:

-Que $C_{i}$ seja inferior ou igual a $C_{j}(i \leq j)$; e

- Que $C_{i}$ seja inferior a $C_{j}$ quando $a$ é incomparável com todos os limites entre $C_{i}$ e $C_{j}\left(a R b_{i}, \forall f\right.$, tal que $\left.i<f \leq j\right)$.

Em suma:

- Quando as avaliações de uma alternativa forem entre os dois limites de uma classe em cada critério, então, ambos os procedimentos classificam esta alternativa para esta classe; e

-Uma divergência existe entre os resultados dos dois procedimentos de classificação somente quando uma alternativa é incomparável para um ou vários limites; em tais casos, a regra de classificação pessimista classifica a alternativa na classe mais inferior que a otimista classifica. 